\title{
Circulating Von Willebrand factor and high molecular weight multimers as markers of endothelial injury predict COVID-19 in-hospital mortality
}

\author{
Aurélien Philippe ${ }^{1} \cdot$ Richard Chocron $^{2} \cdot$ Nicolas Gendron $^{1} \cdot$ Olivier Bory $^{3} \cdot$ Agathe Beauvais $^{3} \cdot$ Nicolas Peron $^{4}$. \\ Lina Khider ${ }^{5}$. Coralie L. Guerin ${ }^{6} \cdot$ Guillaume Goudot $^{5} \cdot$ Françoise Levasseur $^{15}$. Christophe Peronino ${ }^{1}$. \\ Jerome Duchemin $^{13}$. Julie Brichet ${ }^{1}$. Elise Sourdeau ${ }^{14}$. Florence Desvard ${ }^{1}$. Sébastien Bertil ${ }^{1}$. Frédéric Pene ${ }^{16}$. \\ Cherifa Cheurfa ${ }^{17}$. Tali-Anne Szwebel $^{18}$. Benjamin Planquette ${ }^{7}$. Nadia Rivet ${ }^{1}$. Georges Jourdi ${ }^{8}$. \\ Caroline Hauw-Berlemont ${ }^{4}$. Bertrand Hermann ${ }^{4} \cdot$ Pascale Gaussem $^{1} \cdot$ Tristan Mirault $^{9}$ - Benjamin Terrier ${ }^{10}$. \\ Olivier Sanchez ${ }^{7}$ Jean-Luc Diehll ${ }^{11}$. Michaela Fontenay ${ }^{12}$. David M. Smadja ${ }^{1} \mathbb{C}$
}

Received: 3 December 2020 / Accepted: 14 December 2020 / Published online: 15 January 2021

○) The Author(s), under exclusive licence to Springer Nature B.V. part of Springer Nature 2021

\begin{abstract}
Background Coronavirus disease 2019 (COVID-19) is a respiratory disease associated with endotheliitis and microthrombosis.

Objectives To correlate endothelial dysfunction to in-hospital mortality in a bi-centric cohort of COVID-19 adult patients. Methods Consecutive ambulatory and hospitalized patients with laboratory-confirmed COVID-19 were enrolled. A panel of endothelial biomarkers and von Willebrand factor (VWF) multimers were measured in each patient $\leq 48 \mathrm{~h}$ following admission.

Results Study enrolled 208 COVID-19 patients of whom 23 were mild outpatients and 189 patients hospitalized after admission. Most of endothelial biomarkers tested were found increased in the 89 critical patients transferred to intensive care unit. However, only von Willebrand factor antigen (VWF:Ag) scaled according to clinical severity, with levels significantly higher in critical patients (median 507\%, IQR 428-596) compared to non-critical patients $(288 \%, 230-350, p<0.0001)$ or COVID19 outpatients $(144 \%, 133-198, p=0.007)$. Moreover, VWF high molecular weight multimers (HMWM) were significantly higher in critical patients (median ratio 1.18, IQR 0.86-1.09) compared to non-critical patients $(0.96,1.04-1.39, p<0.001)$. Among all endothelial biomarkers measured, ROC curve analysis identified a VWF:Ag cut-off of $423 \%$ as the best predictor for in-hospital mortality. The accuracy of VWF:Ag was further confirmed in a Kaplan-Meier estimator analysis and a Cox proportional Hazard model adjusted on age, BMI, C-reactive protein and D-dimer levels.

Conclusion VWF:Ag is a relevant predictive factor for in-hospital mortality in COVID-19 patients. More than a biomarker, we hypothesize that VWF, including excess of HMWM forms, drives microthrombosis in COVID-19.
\end{abstract}

Keywords Endothelial activation $\cdot$ COVID-19 $\cdot$ Von Willebrand factor $\cdot$ Multimers $\cdot$ Mortality $\cdot$ Microthrombosis

\section{Introduction}

Coronavirus disease 2019 (COVID-19) is a respiratory disease caused by the severe acute respiratory syndrome coronavirus 2 (SARS-CoV-2) characterized by an intense inflammatory syndrome associated with a coagulopathy

David M. Smadja

david.smadja@aphp.fr

Extended author information available on the last page of the article and has been described as an endothelial disease $[1,2]$. Increased D-dimer has been proved to be the most relevant biomarker of the COVID-19-associated coagulopathy [3]. Indeed, a high D-dimer level at the time of patients admission has been reported to be associated with the COVID-19 diagnostic likelihood [4], disease severity, risk of intensive care unit (ICU) referral and mortality [5, 6]. Moreover, we recently proposed that in COVID-19, D-dimer levels reflect pulmonary microvascular thrombosis at the origin of right ventricle dysfunction [7]. Autopsy case series confirmed the hypothesis that both endothelial inflammation 
and microvascular thrombosis are prominent in the pulmonary, renal and intestinal vasculature. This microthrombotic process has been described as closely related to endothelial lesions characterized by endotheliitis [8], abnormal angiogenesis in lungs [9] and also an increase in circulating endothelial cells (CECs) in COVID-19 patients $[4,10]$. This endothelial injury is probably the result of a combination of SARS-CoV-2 direct entry in endothelial cells (ECs) [8], the subsequent prothrombotic phenotype of ECs and the surrounding tissue collateral damage secondary to cytokine release and complement-system activation [11, 12]. In line, we recently described an association between plasma angiopoietin-2 (Ang2) levels at admission and COVID19 patients referral to the ICU [13]. However, the precise pathogenesis of endothelial injury and its deleterious consequences in COVID-19 are still elusive and a better understanding of these processes could help physicians, especially in the ICU, to determine the appropriate therapeutic strategies for preventing and treating coagulopathy associated with endothelial damage.

The objective of the present study was to assess to what extent endothelial activation biomarkers measured at admission were predictive of in-hospital mortality in a large cohort of 208 adult COVID-19 patients.

\section{Materials and methods}

\section{Study design and population}

We performed a bi-centric cross-sectional study of adult ( $\geq 18$-years old) COVID-19 hospitalized and ambulatory patients in two French hospitals (European Georges Pompidou Hospital and Cochin-Hotel Dieu Hospital, Paris, France) between March 13 and June 26, 2020. The study was performed in accordance with the Declaration of Helsinki and a written consent form was signed by all patients included or their trusted relatives at the time of enrollment (SARCODO 2020-A01048-31A, NCT04624997). All included patients, hospitalized or not, presented a confirmed diagnosis of COVID-19, using a reverse transcriptase-polymerase chain reaction (RT-PCR) assay on nasopharyngeal swab samples (Allplex ${ }^{\mathrm{TM}}$ 2019-nCoV Assay, Seegene, Seoul, South Korea) as previously described [14]. Data were automatically analyzed using Seegene viewer software.

Patients were classified according to World Health Organization guidance (WHO) as non-critical (median oxygen requirement $3 \mathrm{l} / \mathrm{min}$; WHO score range 4-7) or critical (requiring mechanical ventilation, WHO score range 8-9) in the first $48 \mathrm{~h}$ following admission for clinically suspected COVID-19. Outpatients were COVID-19 patients who met no hospitalization criteria and returned home immediately after RT-PCR testing for COVID-19 diagnosis. None of the outpatients required supplemental oxygen, were later hospitalized or died in the month following COVID-19 diagnosis. Finally, we also included 29 non-COVID-19 non-hospitalized individuals who served as controls. These patients had an initial clinical suspicion of COVID-19, but with mild clinical presentation and a negative RT-PCR result.

Patient characteristics including age, sex, comorbidities, medical history and treatment at admission were recorded. The primary outcome was COVID-19 in-hospital mortality.

\section{Laboratory procedures on admission}

Routine laboratory tests and sampling for an extensive panel of endothelial activation biomarkers were all performed at hospital admission i.e. in the first $48 \mathrm{~h}$ following the admission for suspected COVID-19. Venous blood was collected from patients and controls and processed according to standard laboratory techniques. Routine laboratory tests were plasma creatinine, C-reactive protein (CRP) and highsensitivity cardiac troponin I (Hs-cTnI) conducted on a DXI analyzer (Beckman Coulter, Brea, CA). Regarding coagulation assays and endothelial biomarker measurements, blood was collected in $0.129 \mathrm{M}$ trisodium citrate tubes (9NC BD Vacutainer, Plymouth, UK). Platelet-poor plasma (PPP) was obtained after centrifugation twice at $2500 \times g$ for $15 \mathrm{~min}$ and stored at $-80{ }^{\circ} \mathrm{C}$ until analysis. Measurement of D-dimer was performed using the Vidas D-dimers ${ }^{\circledR}$ assay (Biomérieux, Marcy-Etoile, France) according to the manufacturer's instruction. The plasma concentrations of soluble E-selectin (sE-sel), soluble endoglin (sEng), angiopoietin-1 (Ang1), Ang2 and vascular cell adhesion protein 1 (VCAM-1) were quantified in PPP using a Human Magnetic Luminex Assay (R\&D Systems, Minneapolis, MN). Data were assessed with the Bio-Plex 200 using the Bio-Plex Manager 5.0 software (Bio-Rad, Marnes-la-Coquette, France). Soluble thrombomodulin (sTM, DTHBD0, R\&D Systems) and soluble endothelial protein C receptor (sEPCR, 00264, Diagnostica Stago, Asnières, France) were measured using ELISAs on plasma samples according to the manufacturer's recommendations.

CECs were isolated and counted by immunomagnetic separation using anti-CD146 monoclonal-antibody-coated beads and marking reagent acridin orange as previously described [4].

Von Willebrand factor antigen (VWF:Ag) and activity (VWF:Rco) used (STA Liatest, Diagnostica Stago) and a latex immunoturbidimetric assay (vWF:Rco; Diagnostica Stago), respectively, on a STA-R® Max coagulometer (Diagnostica Stago). The VWF multimers pattern was evaluated by a semi-automated assay: the Hydragel 5 von Willebrand multimers kit, on the Hydrasis 2 Scan instrumentation (Sebia, Lisses, France) [15, 16]. All samples were defrozen in a water bath at $37^{\circ} \mathrm{C}$ for $5 \mathrm{~min}$, were treated with 
the appropriate sample diluent and incubated for $20 \mathrm{~min}$ at $45{ }^{\circ} \mathrm{C}$. The dilution ratio was adapted to adjust all patients' samples to $100 \%$ VWF:Ag. The treated plasma samples were loaded onto Hydragel 5 von Willebrand multimers gels $(5 \mu \mathrm{l} /$ well) for migration by agarose gel electrophoresis. Direct immunofixation by anti-VWF antibodies and visualization by peroxidase-labeled antibodies revealed the multimeric profile. According to a standard VWF multimers evaluation [17], the relative proportions of low- $(\leq 5$-mers, LMWM), intermediate- (6- to 10-mers, IMWM), and high-molecular-weight multimers (>10-mers, HMWM) of VWF were determined using densitometry analysis on a Hydrasis 2 Scan. Multimers pattern curves were displayed on the Sebia Phoresis software. For each patient, LMWM, IMWM and HMWM were either expressed as a proportion (expressed in percentage) of each patient's total multimers or as a ratio between patient's and healthy volunteers' pooled plasma.

\section{Statistical analysis}

Continuous data were expressed as median (interquartile range (IQR)] and categorical data as proportions. In the univariate analysis, patients were compared according to COVID-19 status (non-COVID-19 vs COVID-19) and COVID-19 severity (non-critical vs critical) using the Mann-Whitney test and Fisher's exact test for continuous and categorical variables, respectively. The association between levels of endothelial activation biomarkers and COVID-19 severity was assessed using the Kruskal-Wallis and Cochran-Armitage tests for trends for continuous and categorical variables (multiple groups), respectively. In order to estimate the ability of VWF:Ag to predict inhospital mortality, we used receiver operator characteristics (ROC) analysis. We estimated the area under the curve (AUC) and its 95\% confidence interval (CI) and selected the optimal cutoff that illustrated the prognostic ability of VWF:Ag.

In the multivariate analysis, we assessed the association between VWF:Ag and in-hospital mortality using the logistic regression model adjusted for age, body mass index (BMI), D-dimer and CRP levels.

For the survival analysis among patients hospitalized for COVID19, the start of the study was triggered by the diagnosis of SARS-CoV-2 infection. The end of the study was defined either by patient's death during their hospitalization or by discharge alive from the hospital. We used the Kaplan-Meier curve to estimate the survival function from diagnosis to in-hospital death according to the optimal cutoff of VWF:Ag. Survival curves were compared using the logrank test. We used the Cox proportional hazard (PH) model adjusted for age, BMI, D-dimer and CRP levels to investigate the relationships between the increase in VWF:Ag (over the calculated cut-off value) and in-hospital mortality.
All analyses were two-sided and a $p$-value of $<0.05$ was considered to be statistically significant. Statistical analysis was performed using $\mathrm{R}$ studio software including $\mathrm{R}$ version 3.6.3 (RStudio, PBC, Boston, MA).

\section{Results}

\section{Study population}

Between March 13 and June 26, 2020, a total of 208 COVID-19 adult patients comprising 23 outpatients and 185 hospitalized patients were included in this study. Among hospitalized patients at admission, 89 (48.1\%) suffering from critical forms of COVID-19 were under mechanical ventilation (MV) in the ICU, whereas 96 (51.9\%) were hospitalized in medical wards. Among the patients included, $129(62.0 \%)$ were male. The non-COVID-19 control group comprised 17 (58.6\%) females and $12(41.4 \%)$ males. The median age was 39 (IQR 32.0-46.0) in the non-COVID-19 group and 62 (50.0-72.0) in COVID-19 patients. Patients were significantly older $(p<0.001)$ and included a higher proportion of males $(p<0.001)$ than non-COVID-19 patients (Table 1). Compared with the non-COVID-19 group, cardiovascular risk factors were more frequent in COVID-19 patients, especially in those who were hospitalized. Indeed, 105 (50.5\%) COVID-19 patients suffered from hypertension, while obesity, hyperlipidemia and diabetes were found in approximately one third of them. Concerning hospitalized COVID-19 patients, critical and non-critical patients did not significantly differ for most clinical and biological characteristics; however, critical patients had a higher median BMI (28.1, IQR 26.0-33.7) than non-critical patients (24.4, $23.2-28.5, p<0.001)$. Furthermore, critical patients had significantly higher D-dimer, Hs-cTnI and CRP levels $(p<0.001$ for each) than non-critical patients.

\section{Endothelial activation biomarkers are associated with COVID-19 severity}

First, CECs were significantly increased in critical (median, 32.0, IQR 17.0-55.5) COVID-19 patients compared with non-critical patients $(15.0,9.00-28.50, p<0.001)$ (Fig. 1a, Table 2). In contrast, Ang1, sEng and sEPCR levels were not significantly different between COVID-19 and nonCOVID-19 patients (Fig. 1b-d). Compared to the nonCOVID-19 group, levels of sVCAM-1 were increased significantly in non-critical and critical COVID-19 patients without no significant difference between these two groups (critical: median $2935 \mathrm{ng} / \mathrm{ml}$ IQR 1830-5646, non-critical: $2272 \mathrm{ng} / \mathrm{ml}, 1068-4239, p=0.77$ ) (Fig. 1e). Levels of sE-sel (critical: median 42879 pg/ml, IQR 32136-65398, non-critical: $22453 \mathrm{pg} / \mathrm{ml}, 17873-28643, p<0.0001)$, sTM 
Table 1 Demographic, clinical and biological characteristics of COVID-19 and non COVID-19 patients at admission

\begin{tabular}{|c|c|c|c|c|c|}
\hline & \multirow[t]{2}{*}{ Non-COVID-19 $(n=29)$} & \multicolumn{3}{|l|}{ COVID-19 patients } & \multirow[t]{2}{*}{$p$-value } \\
\hline & & Outpatients $(n=23)$ & Non-critical $(n=96)$ & Critical $(n=89)$ & \\
\hline Male sex- $n(\%)$ & $12(41.4)$ & $9(39.1)$ & $55(57.3)$ & $65(73.0)$ & 0.002 \\
\hline Age—years median (IQR) & $39.0[32.0-46.0]$ & $40.0[34.0-46.5]$ & $65.5[55.0-76.0]$ & $62.0[51.0-71.0]$ & $<0.001$ \\
\hline $\mathrm{BMI}-\mathrm{kg} / \mathrm{m}^{2}$ median (IQR) & $24.5[22.1-28.1]$ & $23.3[21.6-24.6]$ & $24.4[23.2-28.5]$ & $28.1[26.0-33.7]$ & $<0.001$ \\
\hline $\begin{array}{l}\text { Delay between first symptoms and hospi- } \\
\text { talization—-days median (IQR) }\end{array}$ & - & - & $7.0[5.0-10.3]$ & $7.0[5.0-10.0]$ & 0.77 \\
\hline \multicolumn{6}{|l|}{ Comorbidities } \\
\hline Obesity-n (\%) & $5(17.2)$ & $0(0.0)$ & $18(18.8)$ & $33(37.1)$ & $<0.001$ \\
\hline Hypertension $-n(\%)$ & $4(13.8)$ & $2(8.7)$ & $53(55.2)$ & $50(56.2)$ & $<0.001$ \\
\hline Hyperlipidaemia $-n(\%)$ & $0(0.0)$ & $2(8.7)$ & $21(21.9)$ & $30(33.7)$ & $<0.001$ \\
\hline Diabetes- $n(\%)$ & $1(3.4)$ & $0(0.0)$ & $23(24.0)$ & $31(34.8)$ & $<0.001$ \\
\hline Chronic kidney disease $-n(\%)$ & $1(3.4)$ & $0(0.0)$ & $9(9.4)$ & $13(14.6)$ & 0.10 \\
\hline Active or history of malignancy- $n(\%)$ & $0(0.0)$ & $2(8.7)$ & $18(18.8)$ & $11(12.4)$ & 0.057 \\
\hline $\begin{array}{l}\text { Coronary artery disease or myocardial } \\
\text { infarction- } n(\%)\end{array}$ & $2(6.9)$ & $1(4.3)$ & $9(9.4)$ & $8(9.0)$ & 0.35 \\
\hline $\begin{array}{l}\text { Stroke or transient ischaemic attack- } n \\
(\%)\end{array}$ & $1(3.4)$ & $0(0.0)$ & $3(3.1)$ & $7(7.9)$ & 0.28 \\
\hline Asthma $-n(\%)$ & $1(3.4)$ & $3(13.0)$ & $8(8.3)$ & $3(3.4)$ & 0.25 \\
\hline History of VTE- $n(\%)$ & $0(0.0)$ & $0(0.0)$ & $3(3.1)$ & $8(9.0)$ & 0.52 \\
\hline \multicolumn{6}{|l|}{ Biological parameters } \\
\hline D-dimer-ng/ml median [IQR] & $214[172-291]$ & $295[140-438]$ & 1089 [798-1889] & $4186[2498-7292]$ & $<0.001$ \\
\hline $\mathrm{CRP}-\mathrm{mg} / \mathrm{l}$ median $[\mathrm{IQR}]$ & - & $28.0[14.8-33.4]$ & $63.6[32.0-117.9]$ & $189.1[121.5-259.9]$ & $<0.001$ \\
\hline Plasma creatinine - $\mu \mathrm{mol} / \mathrm{l}$ median $[\mathrm{IQR}]$ & - & $64.00[64.0-64.0]$ & $68.0[56.5-83.5]$ & $102.0[72.6-214.3]$ & $<0.001$ \\
\hline Hs-cTnI—ng/l median [IQR] & $6.8[5.6-9.1]$ & $7.2[5.6-8.8]$ & $8.1[5.0-15.1]$ & $28.80[15.3-51.7]$ & $<0.001$ \\
\hline \multicolumn{6}{|l|}{ Outcomes } \\
\hline $\mathrm{MV}-n(\%)$ & $0(0.0)$ & $0(0.0)$ & $4(4.2)$ & $89(100.0)$ & $<0.001$ \\
\hline In-hospital mortality- $n(\%)$ & $0(0.0)$ & $0(0.0)$ & $2(2.1)$ & $39(43.8)$ & $<0.001$ \\
\hline $\begin{array}{l}\text { Length of hospitalization—days median } \\
\text { [IQR] }\end{array}$ & - & - & $8.5[5.0-16.0]$ & $26.0[15.0-39.0]$ & $<0.001$ \\
\hline
\end{tabular}

$I Q R$ interquartile range, $B M I$ body mass index, $C R P$ C-reactive protein, VTE venous thromboembolism, $H s$ - $c T n I$ high-sensitivity cardiac troponin I, $M V$ mechanical ventilation obesity was defined as BMI $>30 \mathrm{~kg} / \mathrm{m}^{2}$

(critical: median $636 \mathrm{pg} / \mathrm{ml}$, IQR 499-871, non-critical: $343 \mathrm{pg} / \mathrm{ml}, 298-412, p<0.0001$ ), Ang2 (critical: median $5360 \mathrm{pg} / \mathrm{ml}$, IQR 4244-9776, non-critical: $2022 \mathrm{pg} / \mathrm{ml}$, $1363-2663, p<0.0001$ ), were significantly increased only in critical COVID-19 patients. In outpatients and non-critical COVID-19 patients in medical wards, circulating levels of these endothelial biomarkers circulating levels did not significantly differ from non-COVID-19 patients. (Fig. 1f-h).

In contrast to the abovementioned biomarkers, VWF:Ag levels scaled along with COVID-19 severity. Levels of VWF:Ag levels were significantly higher in all COVID-19 patients (median 367\%, IQR 250-487) compared with nonCOVID-19 patients $(113 \%, 91-152, p<0.0001)$. Furthermore, VWF:Ag levels were significantly higher in critical patients (median 507\%, IQR 428-596) in contrast to noncritical patients $(288 \%, 230-350, p<0.0001)$ and were significantly higher in non-critical patients $(288 \%, 230-350)$ in contrast to COVID-19 outpatients $(144 \%, 133-198$, $p=0.007$, Fig. 1i). In terms of the association of endothelial markers and usual parameters of severity, VWF:Ag levels were the parameter most significantly associated with D-dimer levels $(r=0.794 p<0.0001)$, CRP $(r=0.585$ $p<0.001)$ and troponin I $(r=0.550 p<0.0001)$. As expected, levels of VWF:Ag were strongly correlated with those of VWF:Rco ( $r=0.944, p<0.001$, data not shown).

Following the observation that increased VWF:Ag in COVID-19 patients mirrored clinical severity, we next explored VWF involvement in COVID-19, performing VWF multimer analysis in both randomly generated subgroups of 40 critical and 37 non-critical included patients. No significant difference was observed in IMWM, while a significant decreased in LMWM was observed (Fig. 2a, b). Strikingly, VWF HMWM (ratio) were significantly increased in critical patients (median 1.18, IQR 1.04-1.39) compared to 
A
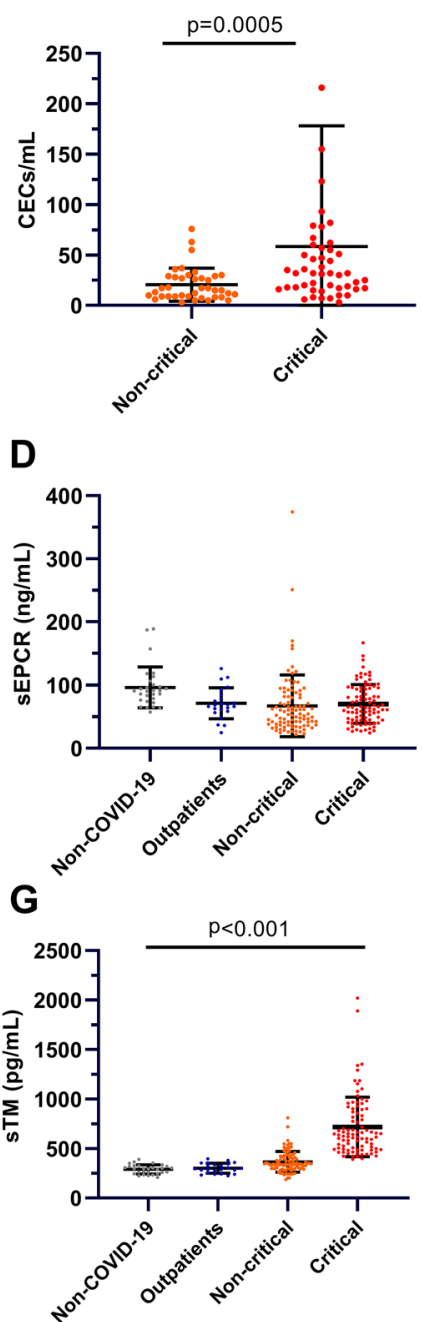

B

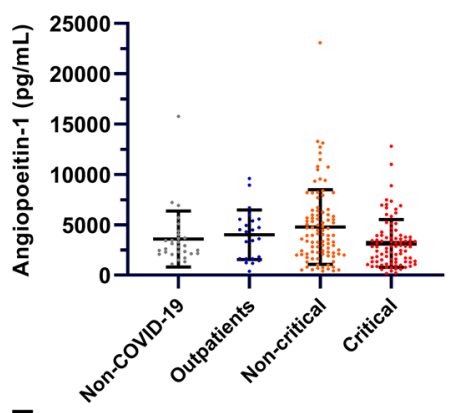

E

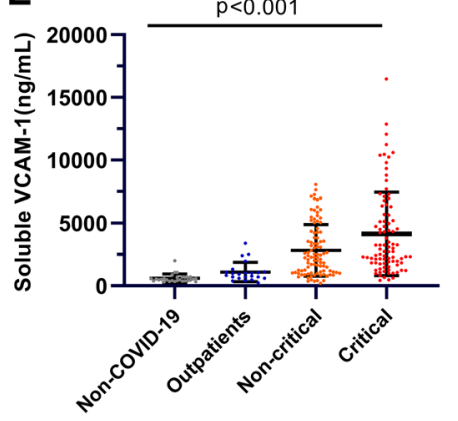

H

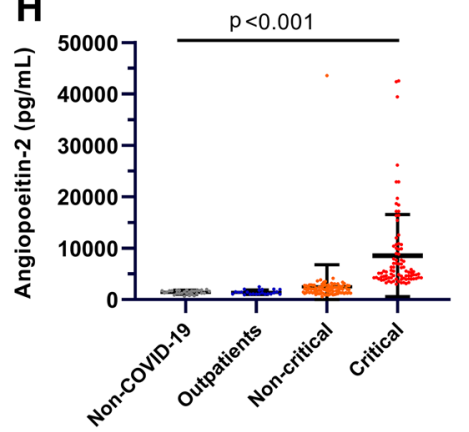

C

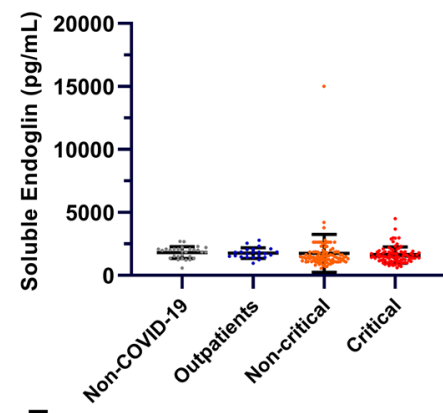

$\mathbf{F}$

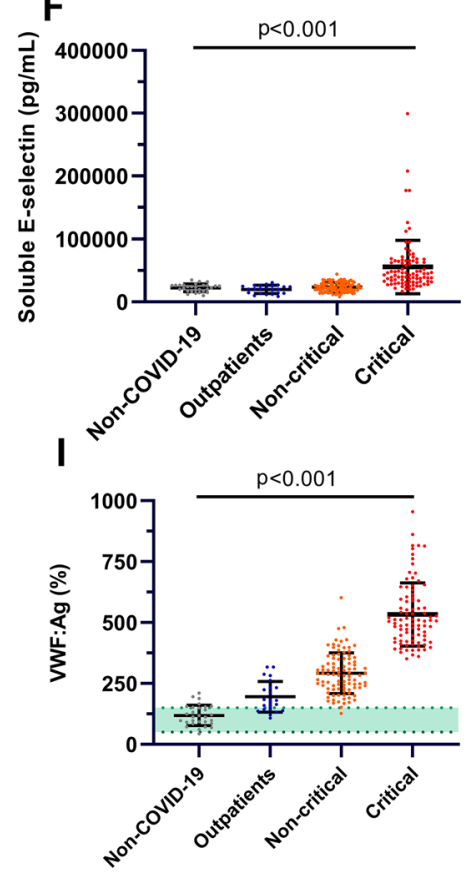

Fig. 1 Levels of endothelial activation biomarkers according to critical patients, non-critical patients, outpatients, and non-COVID-19 individuals. Datapoints indicate individual measurements, whereas horizontal bars represent the means with standard deviations. Green shaded areas indicate the normal ranges of values. $p$-value comes from the Mann-Whitney for comparison between two groups and the Kruskal Wallis test for comparison between the four groups. a Measurement of circulating endothelial cells (CECs) in 37 critical and 40 non-critical COVID-19 patients at admission. Plasma levels of angiopoietin-1 (b), soluble endoglin (c), soluble endothelial protein $\mathrm{C}$ receptor (sEPCR) (d), soluble vascular cell adhesion molecule (VCAM-1) (e), soluble E-selectin (f), sTM (soluble thrombomodulin) (g), angiopoietin-2 (h), von Willebrand factor antigen (VWF:Ag) (i) in 89 critical and 96 non-critical COVID-19 patients, 23 COVID-19 outpatients and 29 non-COVID-19 individuals at admission non-critical patients $(0.96,0.86-1.09, p<0.001$, Fig. 2c, d), in line with the decreased LMWM.

\section{VWF is the best endothelial biomarker to predict in-hospital mortality in COVID-19}

Subsequently, we further investigated the association between the levels of endothelial biomarkers at admission and in-hospital mortality. First, we performed a univariate logistic regression model for all endothelial biomarkers measured. In decreasing order, variations in levels of HMWM (ratio) (odds ratio, OR 116, 95\% CI 10.2-1943, $p<0.001$ ), HMWM (\%) (OR 1.11, 95\% CI 1.00-1.24, $p=0.048), \mathrm{vWF}: \mathrm{Ag}(\mathrm{OR} 1.01,95 \%$ CI 1.01-1.02, $p<0.001)$ and sTM (OR 1.01, 95\% CI 1.01-1.01, $p<0.001)$ were the most significantly associated with in-hospital mortality (Table 2). In order to evaluate the respective discriminatory ability between survivors and non survivors of those four biomarkers, we generated ROC curves. Among generated ROC curves in hospitalized patients, VWF:Ag (AUC 0.92, 95\% CI 0.88-0.96) proved to be the best predictive parameter followed by sTM $(0.91,95 \%$ CI $0.87-0.95)$, HMWM (as a ratio vs normal plasma: $0.77,95 \%$ CI $0.64-0.90$, as a percentage $0.64,95 \%$ CI $0.49-0.79$ ) (Fig. 3). Therefore, we 
Table 2 Admission level and univariate outcome association of endothelial biomarkers and von Willebrand factor-related parameters of COVID19 and non-COVID-19 patients

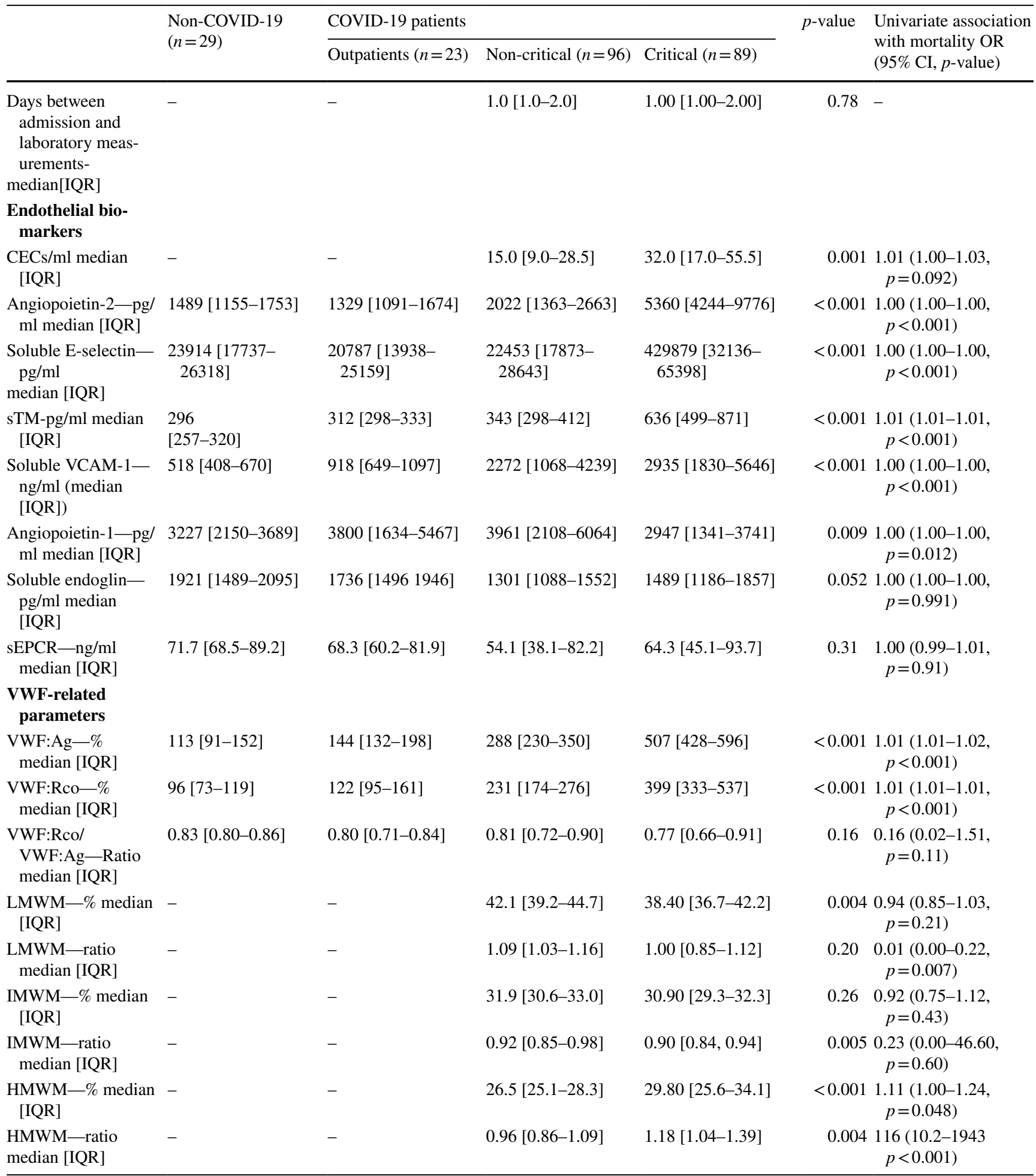

$I Q R$ interquartile range, $O R$ odds ratio, $C I$ confidence interval, $C E C s$ circulating endothelial cells, $s T M$ soluble thrombomodulin, $V C A M-1$ vascular cell adhesion molecule 1, $s E P C R$ soluble endothelial protein $\mathrm{C}$ receptor, $V W F: A g$ von Willebrand factor antigen, $\nu W F: R c o$ von Willebrand factor ristocetin cofactor activity, $L M W M$ von Willebrand factor low-molecular weight multimers, $I M W M$ von Willebrand factor intermediatemolecular weight multimers, $H M W M$ von Willebrand factor high-molecular weight multimers 
used the ROC curve of VWF:Ag to estimate a cut-off value to predict in-hospital mortality. A VWF:Ag level at $423 \%$ provided an optimal sensitivity-specificity balance with an acceptable sensitivity of $95.1 \%$ (95\% CI 88.0-99.1) associated with an excellent negative predictive value of $98.7 \%$ (95\% CI 95.1-99.8) to predict in-hospital mortality.

Indeed, in a univariable analysis model a VWF:Ag level over $423 \%$ at admission was significantly associated with higher in-hospital mortality (OR 89.7 95\% CI 25.9-567.4, $p<0.001)$. This association remained significant in a multivariable analysis model adjusted on age, BMI, D-dimer and CRP (OR 25.6, 95\% CI 5.6-198.2, $p<0.001$ ) (Table 3).

This ability of the vWF:Ag cut-off to predict in-hospital mortality was additionally validated in a Kaplan-Meier estimator ( $p<0.001$, Fig. 4 ) and a Cox proportional hazard analysis adjusted for age, BMI, D-dimer and CRP (Hazard ratio, HR 9.46, 95\% CI 1.99-44.9, $p=0.005$, Fig. 5).

\section{Discussion}

In this study, we demonstrated that markers of endothelial injury in COVID-19 patients were related to severity of patients at admission and to in-hospital mortality. To our knowledge, this study is the first one to examine an extensive panel of endothelial circulating biomarkers, including the assessment of circulating endothelial cells, soluble markers and VWF multimers patterns in such a range of COVID-19 severities, from mildly symptomatic outpatients to critically ill patients, in a cohort of this scale and a nonCOVID-19 control group. The best predictive marker of inhospital mortality was found to be VWF:Ag, which makes relevant its evaluation in daily practice during COVID-19 hospitalization.

First of all, we confirmed that an endothelial dysfunction occurs in COVID-19, in particular in severe forms. Previously, our team demonstrated an association between Ang2 levels at admission and ICU referral [13]. Moreover, Goshua et al. showed that STM levels measured in critical COVID19 patients were increased and were a good predictor of in-hospital mortality [18]. Recently, Cugno et al. reported increased levels of sTM and sE-sel in severe COVID-19 patients without establishing their relationship to in-hospital mortality [19]. In the present study, we found an increase in several specific biomarkers of EC injury in COVID19 patients compared with non-COVID-19 individuals, the most striking differences being observed in VWF:Ag. Notably, we identified two distinctive profiles of biomarkers according to clinical severity. Indeed, Ang2, sE-sel and sTM were elevated only in critical patients, whereas only VWF:Ag increased accordingly to disease severity. Von Willebrand factor, which mediates platelet adhesion and aggregation through its binding to platelet GPIX-Ib receptor, is contained in endothelial Weibel-Palade bodies (WBPs), mainly in the form of large multimers [20]. Inflammatory cytokines, in particular interleukin 1 and tumor necrosis factor $\alpha$, were shown to trigger endothelium activation which results notably into the release of endothelial WBPs content and therefore VWF by exocytosis [21]. As expected, in our cohort, VWF:Ag levels correlated with inflammatory markers such as CRP. To date, the only therapy which has demonstrated clinical proofs on in-hospital mortality in COVID-19 is steroids, reducing deaths by approximately one-third among critically-ill patients [22]. In addition to well-known systemic anti-inflammatory effects, steroids exert various direct effects on EC. Indeed, steroids inhibit endothelial phenotype induced by inflammation [23-25] and improve endothelial barrier integrity through upregulation of junctional proteins such as occludin, claudin-5, and VE-cadherin [26]. Thus, steroids could decrease excessive endothelium activation and subsequent VWF release which could explain, at least partly, their clinical beneficial in COVID-19. Future studies need to appreciate endothelial lesion markers, in particular VWF, as prognostic marker during steroids treatment. However, in the present study, VWF:Ag remained independently associated with in-hospital mortality after adjustment for inflammatory biomarkers, which supports the hypothesis of additional causes of VWF secretion by ECs. Indeed, endothelium is emerging as a key target organ of SARS-CoV-2 infection. Dissociation between markers of activation, such as sE-sel, and VWF:Ag in critical and non-critical patients could reflect the overwhelming synthesis and release of VWF in pulmonary vasculature due to a combination of inflammation, direct viral destruction of ECs, and also lung tissue hypoxia [27].

In order to deepen the characterization of VWF involvement in COVID-19, we reported increased HMWM in critical compared with non-critical patients. Following an initial endothelial lesion caused by multiple factors, an increase in HMWM of VWF could promote local microthrombosis which itself promotes additional ECs lesion in a vicious cycle. Indeed, under physiological conditions, newly secreted VWF multimers are rapidly cleaved into smaller, less reactive multimers by the metalloproteinase ADAMTS13 during VWF secretion from ECs [28]. ADAMTS13 acquired deficiency also known as thrombotic thrombocytopenic purpura (TTP), is a life-threatening condition well known to induce an accumulation of large VWF multimers which binds spontaneously to platelets, resulting in diffuse microthrombosis [29]. In a small case series, Escher et al. reported normal ADAMTS13 activity in severe COVID-19 patients [30], whereas Rovas et al. found a minor decrease in ADAMTS13 in critically-ill patients but in no way comparable to the nearly total deficiency associated with TTP [31]. Taken together, our results suggest a massive release of unprocessed VWF from activated ECs that may 
A

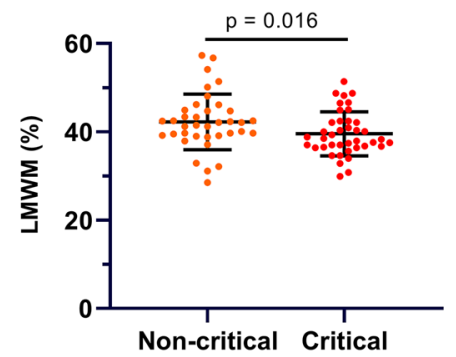

B

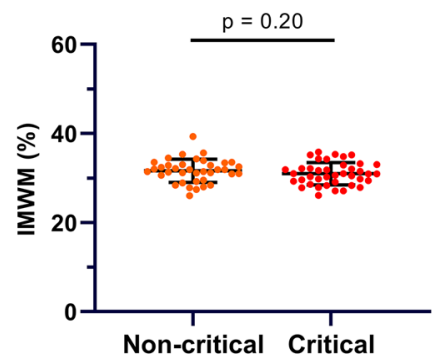

C

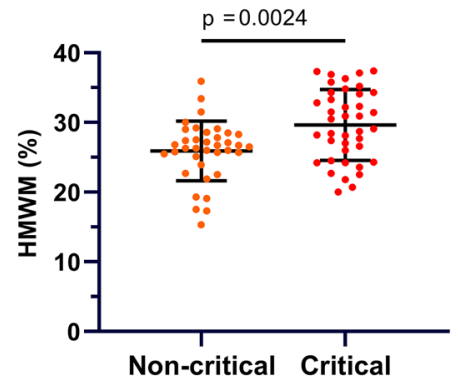

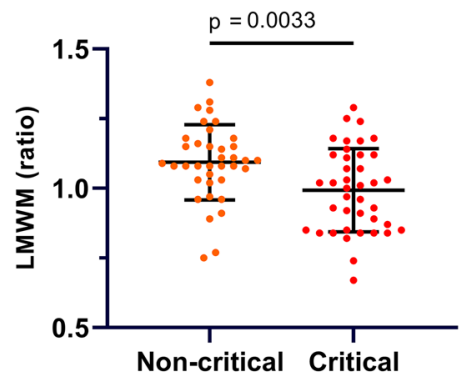
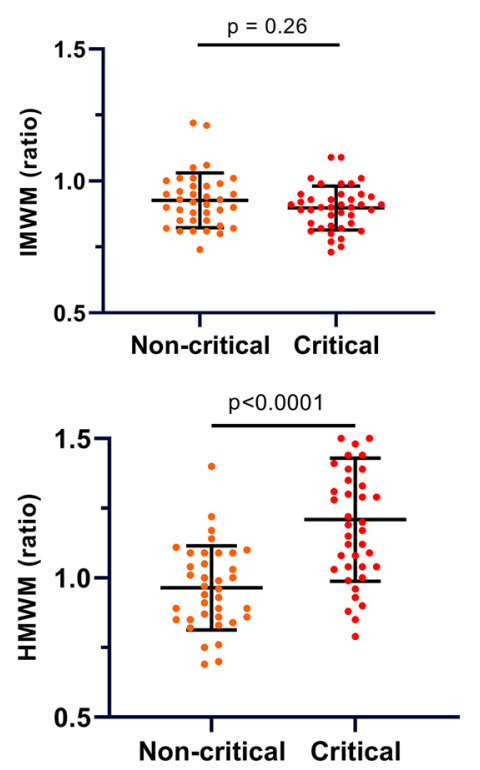

D

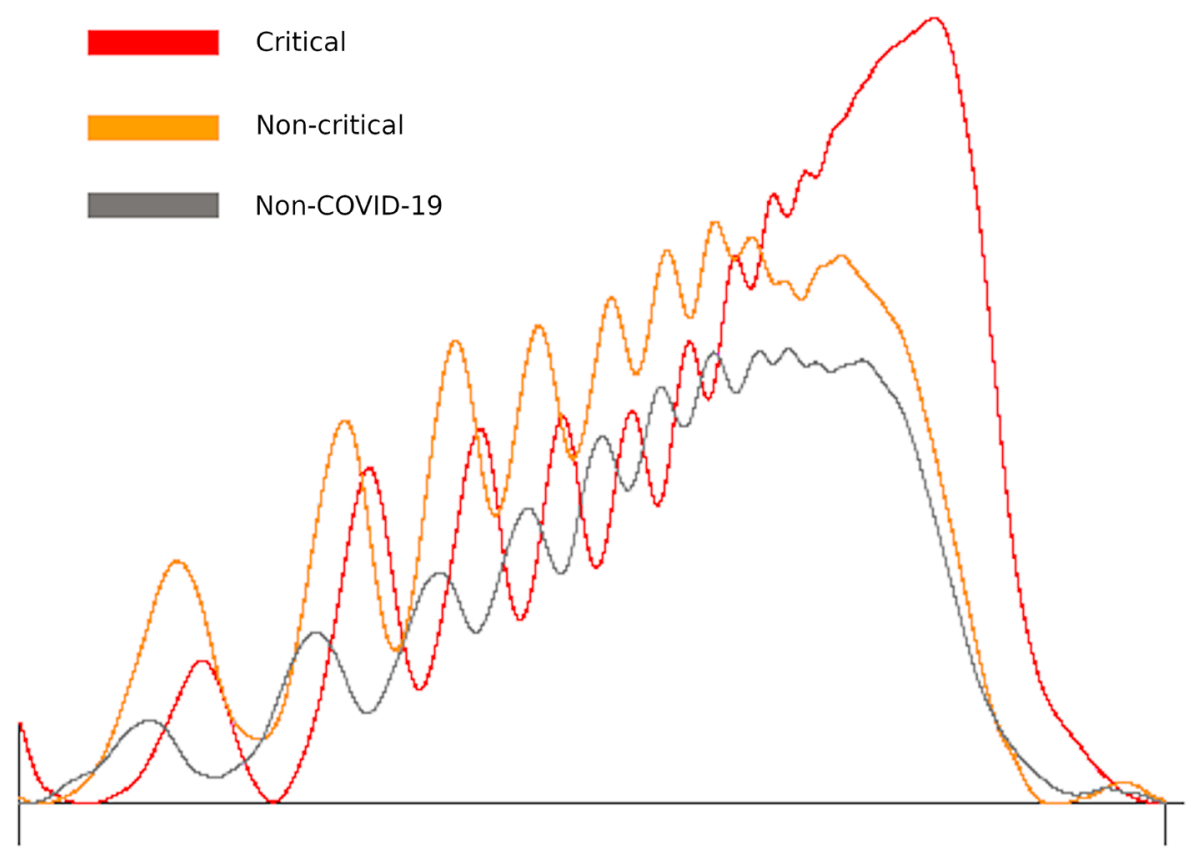


4Fig. 2 Comparisons of von Willebrand factor multimers pattern in 40 critical and 37 non-critical COVID-19 patients. Datapoints indicate individual measurements, whereas horizontal bars show mean with standard deviation. P-value comes from the Mann-Whitney test for comparison between the two groups. a Low molecular weight multimers (LMWM) expressed as a percentage of total multimer (left panel) or a ratio compared to healthy individuals' pool of plasma (right panel). b Intermediate molecular weight multimers (IMWM) expressed as a percentage of total multimers (left panel) or a ratio compared to healthy individuals' pool of plasma (right panel). c High molecular weight multimers (HMWM) expressed as a percentage of total multimers (left panel) or a ratio compared with healthy individuals' pool of plasma (right panel). d Curves showing Von Willebrand factor multimers pattern analysed in densitometric analysis of one critical COVID-19 patient, one non-critical COVID-19 patient and one non-COVID-19 individual. HMWM are located at the right part of each curves. (Refer to materials and methods for detailed multimers classification)

overwhelm the enzymatic capacity of ADAMTS13 rather than a true ADAMTS13 deficiency. In COVID-19, this link between VWF and pulmonary microthrombosis requires further confirmation. A way to highlight this link could be measuring VWF:Ag at multiple time points during the hospitalization of severe COVID-19 patients and evaluate its correlation with advanced respiratory monitoring parameters established to mirror the pulmonary microcirculation dysfunction such as pulmonary physiological dead space and its sub-components airway dead-space and alveolar dead-space $[32,33]$.

Regarding clinical practice, in light of our finding that VWF:Ag levels at the time of COVID-19 patients' aggravation (approximated in this study by hospitalization) might predict in-hospital mortality, we recommend including systematic VWF:Ag measurement into the monitoring strategy of all COVID-19 patients. In addition, it will be very important to investigate VWF:Ag follow-up, which could provide additional prognostic information both in the critical care and in the non-critical care settings. This strategy should be all the easier to set up, given that VWF:Ag is a common analysis in hematology laboratory in most healthcare centers worldwide [34].

Furthermore, establishing a well-validated VWF:Ag cut-off could help in identifying patients who might benefit the most from adjunction of antiplatelet therapies to standard of care. Indeed, VWF and, in particular, HMWM, is an essential component in platelet aggregation. Manne et al. and Hottz et al. both highlighted platelet hyperactivity in COVID-19-associated pathophysiology [35, 36]. Aspirin could present a way to hinder microthrombosis in COVID19 and therefore to stop the vicious cycle of endothelial

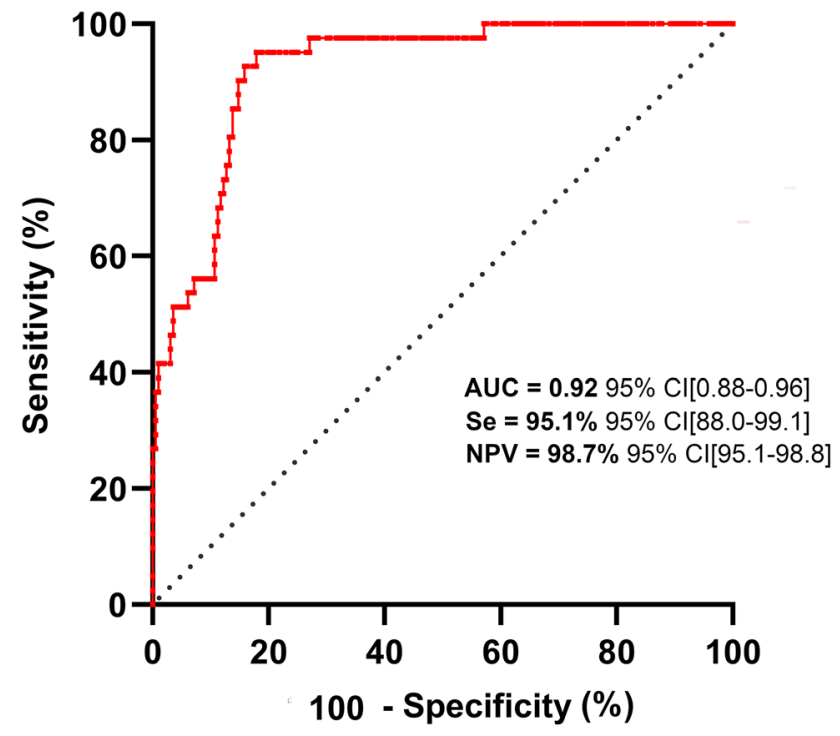

Fig. 3 Receiver operating curves evaluating unadjusted von Willebrand factor antigen's ability to predict in-hospital-mortality. The diagonal black dotted segment is the reference line. AUC area under the curve, $S e$ sensitivity, $N P V$ negative predictive value, $C I$ confidence interval

lesion described above. In the pre-COVID-19 era, several single-center retrospective observational cohort studies assessed a possible association between pre-hospital anti-platelet agent (the vast majority of which is aspirin) therapy and mortality in sepsis or acute respiratory distress syndrome (ARDS). Patients admitted with community-acquired pneumonia on anti-platelet therapy have a lower admission rate to the ICU and shorter hospital stay [37]. In a general population of ICU admissions, patients with pre-admission antiplatelet therapy had a decreased risk of developing ARDS [38, 39] and multi-organ failure [40]. Furthermore, in ICU patients with septic shock or ARDS being treated with antiplatelet drugs, several studies reported a reduction in mortality rate [41-43]. In COVID-19, Chow et al. recently showed in a retrospective observational cohort study of adult patients admitted with COVID-19 that patients who received aspirin within $24 \mathrm{~h}$ of admission or seven days prior to admission displayed a decreased risk of MV, ICU admission and in-hospital mortality with no differences in major bleeding or overt thrombosis between aspirin users and aspirin non-users [44]. Aspirin therefore appears to be a promising treatment in COVID-19, although it should be further evaluated in prospective clinical trials. Another attractive approach to 
Table 3 Unadjusted and adjusted logistic regression analyses of von Willebrand factor antigen levels as a predictive factor for COVID-19 in-hospital mortality

\begin{tabular}{llll}
\hline Logistic regression model with in-hospital mortality as the outcome \\
& & Univariate analysis & \\
& & OR $(95 \%$ CI, $p$-value $)$ & $\begin{array}{l}\text { Multivariate analysis } \\
\text { OR }(95 \% \text { CI, } p \text {-value })\end{array}$ \\
\hline VWF:Ag (\%) & $<423$ & - & - \\
& $>423$ & $89.7(25.9-567.4, p<0.001)$ & $25.6(5.6-198.2, p<0.001)$ \\
Age $(\text { years })^{*}$ & $<59$ & - & - \\
& $>59$ & $2.5(1.2-5.2, p=0.013)$ & $1.6(0.5-4.9, p=0.41)$ \\
BMI $\left(\mathrm{kg} / \mathrm{m}^{2}\right)^{*}$ & $<25$ & - & - \\
& $>25$ & $3.4(1.6-8.2, p=0.004)$ & $0.6(0.1-2.2, p=0.42)$ \\
D-dimer $(\mathrm{ng} / \mathrm{ml})^{*}$ & $<1377$ & - & - \\
& $>1377$ & $28.6(8.5-179.1, p<0.001)$ & $3.6(0.8-26.1, p=0.13)$ \\
CRP $(\mathrm{mg} / \mathrm{l})^{*}$ & $<110$ & - & - \\
& $>110$ & $5.5(2.1-17.2, p=0.001)$ & $1.5(0.4-5.8, p=0.53)$ \\
\hline
\end{tabular}

$B M I$ body mass index, CRP C-reactive protein, $V W F: A g$ von Willebrand factor antigen

In the multivariate analysis the model was adjusted for age*, BMI $\left(25 \mathrm{~kg} / \mathrm{m}^{2}\right.$ as cut-off), D-dimer* and CRP*. *Variables were dichotomized according to the median

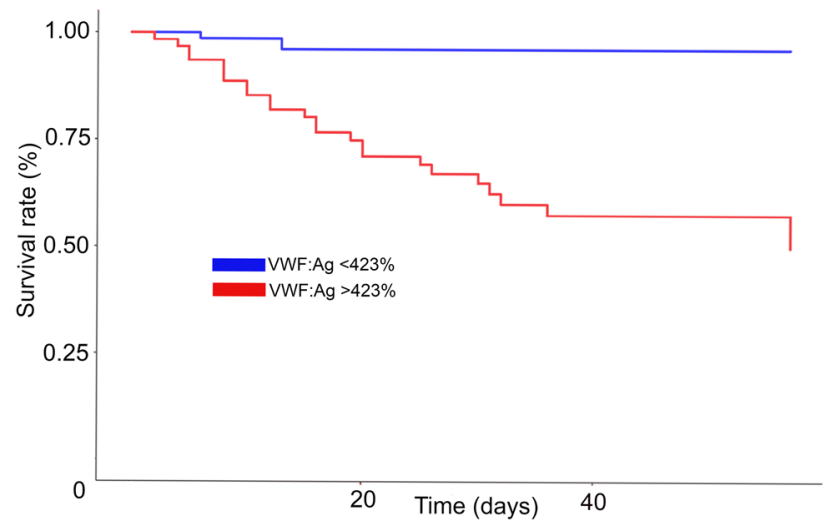

Fig. 4 Survival curves according to von Willebrand factor antigen concentration using a Kaplan-Meier estimator. Data are shown for patients with low von Willebrand factor antigen $(<423 \%)$ and high von Willebrand factor antigen $(>423 \%)$. Survival curves are compared using the log-rank test hamper excessive VWF large multimers binding to platelets and the ensuing microthrombosis could be the use of novel TTP treatments caplacizumab [45] or anfibatide [46], both of which inhibit the binding of platelets to the VWF at the GPIX-Ib receptor.

Limitation of our study include the absence of iterative biomarker measurement over time to provide a more accurate picture of endothelial dysfunction during COVID-19 evolution.

In conclusion, our study provides new insights confirming that COVID-19 is a microvascular disease, in particular for critical forms of COVID-19. Inflammation and SARS-CoV-2 activates and/or directly injure ECs. In our cohort, circulating VWF levels were highly correlated with clinical severity and were the best endothelial marker to predict in-hospital mortality. Beyond its involvement as a biomarker, VWF and HMWM as thrombosis actors

$\begin{array}{lcccc}\text { VWF }: \text { Ag }>423 \% & \text { n } & \text { a HR } & 95 \% \text { CI } & \text { p-value } \\ \text { Age }>59 \text { years } & 74 & 9.46 & 1.99-44.9 & 0.005 \\ & & & & \\ \text { BMI }>25 \mathrm{~kg} / \mathrm{m}^{2} & 111 & 1.30 & 0.55-3.10 & 0.549 \\ & & & & \\ \text { D-dimer }>1377 \mathrm{ng} / \mathrm{mL} & 124 & 0.52 & 0.20-1.40 & 0.163 \\ & & & & \\ \text { CRP }>110 \mathrm{mg} / \mathrm{L} & 117 & 3.56 & 0.80-16.0 & 0.097 \\ & & & & \\ & 96 & 0.79 & 0.28-2.20 & 0.659\end{array}$

Fig. 5 Forest plot showing the Cox proportional hazards model for von Willebrand factor antigen adjusted for age, body mass index, D-dimer and C-reactive protein. Values are hazard ratios with $95 \%$

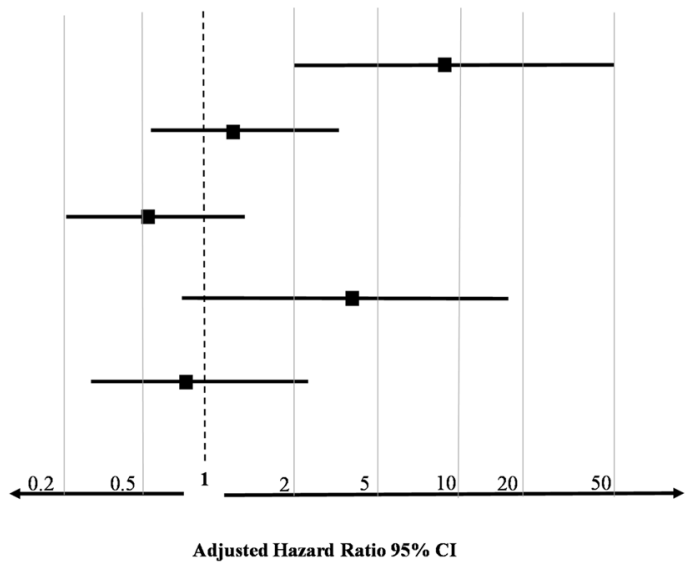

confidence intervals. VWF:Ag von Willebrand factor antigen, $B M I$ body mass index, $C R P$ C-reactive protein, $a H R$ adjusted hazard ratio, $C I$ confidence interval 
emphasize the link between microvascular thrombosis and endothelial injury during COVID-19. Measurement of VWF:Ag could therefore represent a quick, easy, and non-invasive way to point out the most severe form of COVID-19 but also assess treatments efficiency during follow-up.

Funding This work was funded with grants from French national agency for research ANR SARCODO (Fondation de France) and Mécénat Covid AP-HP.

\section{Compliance with ethical standards}

Conflict of interest All authors declare that they have no conflict of interest.

\section{References}

1. Debuc B, Smadja DM (2020) Is COVID-19 a new hematologic disease? Stem Cell Rev Rep 10:15. https://doi.org/10.1007/s1201 5-020-09987-4

2. Libby P, Lüscher T (2020) COVID-19 is, in the end, an endothelial disease. Eur Heart J 41(32):3038-3044

3. Connors JM, Levy JH (2020) COVID-19 and its implications for thrombosis and anticoagulation. Blood 135(23):2033-2040

4. Khider L, Gendron N, Goudot G et al (2020) Curative anticoagulation prevents endothelial lesion in COVID-19 patients. J Thromb Haemost 18:2391

5. Tang N, Bai H, Chen X et al (2020) Anticoagulant treatment is associated with decreased mortality in severe coronavirus disease 2019 patients with coagulopathy. J Thromb Haemost 18:1520

6. Zhou F, Yu T, Du R et al (2020) Clinical course and risk factors for mortality of adult inpatients with COVID-19 in Wuhan, China: a retrospective cohort study. Lancet Lond Engl 395(10229):1054-1062

7. Goudot G, Chocron R, Augy J-L et al (2020) Predictive factor for COVID-19 worsening: insights for high-sensitivity troponin and D-dimer and correlation with right ventricular afterload. Front. Med. 7:20. https://doi.org/10.3389/fmed.2020.586307

8. Varga Z, Flammer AJ, Steiger P et al (2020) Endothelial cell infection and endotheliitis in COVID-19. Lancet Lond Engl 395(10234): 1417-1418

9. Ackermann M, Stark H, Neubert L et al (2020) Morphomolecular motifs of pulmonary neoangiogenesis in interstitial lung diseases. Eur Respir J 55(3):1900933

10. Guervilly C, Burtey S, Sabatier F et al (2020) Circulating endothelial cells as a marker of endothelial injury in severe COVID-19. J. Infect. Dis. 222:1789

11. Magro C, Mulvey JJ, Berlin D et al (2020) Complement associated microvascular injury and thrombosis in the pathogenesis of severe COVID-19 infection: a report of five cases. Transl Res 220:1-13

12. Mehta P, McAuley DF, Brown M et al (2020) COVID-19: consider cytokine storm syndromes and immunosuppression. Lancet Lond Engl 395(10229):1033-1034

13. Smadja DM, Guerin CL, Chocron R et al (2020) Angiopoietin-2 as a marker of endothelial activation is a good predictor factor for intensive care unit admission of COVID-19 patients. Angiogenesis. 23:611
14. Péré H, Podglajen I, Baillard J-L et al (2020) Thermal inactivation and nucleic acid amplification-based testing for SARS-CoV-2. J Clin Virol 131:104588

15. Pikta M, Zemtsovskaja G, Bautista $\mathrm{H}$ et al (2018) Preclinical evaluation of a semi-automated and rapid commercial electrophoresis assay for von Willebrand factor multimers. J. Clin. Lab. Anal. 32(6):22416

16. Bowyer AE, Goodfellow KJ, Seidel H et al (2018) Evaluation of a semi-automated von Willebrand factor multimer assay, the Hydragel 5 von Willebrand multimer, by two European Centers. Res Pract Thromb Haemost 2(4):790-799

17. Veyradier A, Obert B, Houllier A, Meyer D, Girma JP (2001) Specific von Willebrand factor-cleaving protease in thrombotic microangiopathies: a study of 111 cases. Blood 98(6):1765-1772

18. Goshua G, Pine AB, Meizlish ML et al (2020) Endotheliopathy in COVID-19-associated coagulopathy: evidence from a singlecentre, cross-sectional study. Lancet Haematol 7(8):e575-e582

19. Cugno M, Meroni PL, Gualtierotti R et al (2020) Complement activation and endothelial perturbation parallel COVID-19 severity and activity. J Autoimmun 116:102560

20. Lenting PJ, Casari C, Christophe OD, Denis CV (2012) von Willebrand factor: the old, the new and the unknown. J Thromb Haemost JTH 10(12):2428-2437

21. Chen J, Chung DW (2018) Inflammation, von Willebrand factor, and ADAMTS13. Blood 132(2):141-147

22. Ledford $\mathrm{H}$ (2020) Coronavirus breakthrough: dexamethasone is first drug shown to save lives. Nature 582(7813):469

23. Zakkar M, Luong LA, Chaudhury H et al (2011) Dexamethasone arterializes venous endothelial cells by inducing mitogenactivated protein kinase phosphatase-1: a novel antiinflammatory treatment for vein grafts? Circulation 123(5):524-532

24. Gelati M, Corsini E, De Rossi M et al (2002) Methylprednisolone acts on peripheral blood mononuclear cells and endothelium in inhibiting migration phenomena in patients with multiple sclerosis. Arch Neurol 59(5):774-780

25. Dufour A, Corsini E, Gelati M et al (1998) Modulation of ICAM1, VCAM-1 and HLA-DR by cytokines and steroids on HUVECs and human brain endothelial cells. J Neurol Sci 157(2):117-121

26. Blecharz KG, Drenckhahn D, Förster CY (2008) Glucocorticoids increase VE-cadherin expression and cause cytoskeletal rearrangements in murine brain endothelial cEND cells. J Cereb Blood Flow Metab Off J Int Soc Cereb Blood Flow Metab 28(6):1139-1149

27. Mojiri A, Alavi P, Lorenzana Carrillo MA et al (2019) Endothelial cells of different organs exhibit heterogeneity in von Willebrand factor expression in response to hypoxia. Atherosclerosis 282:1-10

28. Ruggeri ZM (2003) Von Willebrand factor, platelets and endothelial cell interactions. J Thromb Haemost JTH 1(7):1335-1342

29. Dong J, Moake JL, Nolasco L et al (2002) ADAMTS-13 rapidly cleaves newly secreted ultralarge von Willebrand factor multimers on the endothelial surface under flowing conditions. Blood 100(12):4033-4039

30. Escher R, Breakey N, Lämmle B (2020) ADAMTS13 activity, von Willebrand factor, factor VIII and D-dimers in COVID-19 inpatients. Thromb Res 192:174-175

31. Rovas A, Osiaevi I, Buscher K et al (2020) Microvascular dysfunction in COVID-19: the MYSTIC study. Angiogenesis 14:1-13

32. Diehl J-L, Peron N, Chocron R et al (2020) Respiratory mechanics and gas exchanges in the early course of COVID-19 ARDS: a hypothesis-generating study. Ann Intensive Care 10(1):95

33. Diehl J-L, Peron N, Philippe A, Smadja DM (2020) Response to Damiani and colleagues. Ann Intensive Care 10:147

34. Hubbard AR, Hamill M, Eikenboom HCJ et al (2012) Standardization of von Willebrand factor propeptide: value assignment 
to the WHO 6th IS factor VIII/von Willebrand factor, plasma (07/316). J Thromb Haemost 10(5):959-960

35. Hottz ED, Azevedo-Quintanilha IG, Palhinha L et al (2020) Platelet activation and platelet-monocyte aggregate formation trigger tissue factor expression in patients with severe COVID-19. Blood 136(11):1330-1341

36. Manne BK, Denorme F, Middleton EA et al (2020) Platelet gene expression and function in patients with COVID-19. Blood 136(11):1317-1329

37. Lösche W, Boettel J, Kabisch B et al (2012) Do aspirin and other antiplatelet drugs reduce the mortality in critically ill patients? Thrombosis 2012:720254

38. Valerio-Rojas JC, Jaffer IJ, Kor DJ, Gajic O, Cartin-Ceba R (2013) Outcomes of severe sepsis and septic shock patients on chronic antiplatelet treatment: a historical cohort study. Crit Care Res Pract 2013:782573

39. Chen W, Janz DR, Bastarache JA et al (2015) Prehospital aspirin use is associated with reduced risk of acute respiratory distress syndrome in critically ill patients: a propensity-adjusted analysis. Crit Care Med 43(4):801-807

40. Harr JN, Moore EE, Johnson J et al (2013) Anti-platelet therapy is associated with decreased transfusion-associated risk of lung dysfunction, multiple organ failure, and mortality in trauma patients. Crit Care Med 41(2):399-404

41. Tsai M-J, Ou S-M, Shih C-J et al (2015) Association of prior antiplatelet agents with mortality in sepsis patients: a nationwide population-based cohort study. Intensive Care Med 41(5):806-813
42. Boyle AJ, Di Gangi S, Hamid UI et al (2015) Aspirin therapy in patients with acute respiratory distress syndrome (ARDS) is associated with reduced intensive care unit mortality: a prospective analysis. Crit Care Lond Engl 19:109

43. Eisen DP, Leder K, Woods RL et al (2020) Effect of aspirin on deaths associated with sepsis in healthy older people (ANTISEPSIS): a randomised, double-blind, placebo-controlled primary prevention trial. Lancet Respir Med 10:20. https://doi.org/10.1016/ S2213-2600(20)30411-2

44. Chow JH, Khanna AK, Kethireddy S et al (2020) Aspirin use is associated with decreased mechanical ventilation, ICU admission, and in-hospital mortality in hospitalized patients with COVID-19. Anesth Analg. Volume Publish Ahead of Print

45. Dutt T, Shaw RJ, Stubbs MJ et al Real-world evidence of caplacizumab use in the management of acute TTP. Blood

46. Zheng L, Mao Y, Abdelgawwad MS et al (2016) Therapeutic efficacy of the platelet glycoprotein Ib antagonist anfibatide in murine models of thrombotic thrombocytopenic purpura. Blood Adv 1:75-83

Publisher's Note Springer Nature remains neutral with regard to jurisdictional claims in published maps and institutional affiliations.

\section{Affiliations}

\section{Aurélien Philippe ${ }^{1} \cdot$ Richard Chocron $^{2} \cdot$ Nicolas Gendron $^{1} \cdot$ Olivier Bory $^{3} \cdot$ Agathe Beauvais $^{3} \cdot$ Nicolas Peron $^{4}$. Lina Khider ${ }^{5}$. Coralie L. Guerin ${ }^{6}$. Guillaume Goudot ${ }^{5}$. Françoise Levasseur ${ }^{15}$. Christophe Peronino ${ }^{1}$. Jerome Duchemin ${ }^{13}$. Julie Brichet ${ }^{1}$. Elise Sourdeau ${ }^{14}$. Florence Desvard ${ }^{1}$. Sébastien Bertil ${ }^{1}$. Frédéric Pene ${ }^{16}$. Cherifa Cheurfa ${ }^{17}$. Tali-Anne Szwebel $^{18}$. Benjamin Planquette ${ }^{7} \cdot$ Nadia Rivet $^{1}$. Georges Jourdi ${ }^{8}$. Caroline Hauw-Berlemont ${ }^{4} \cdot$ Bertrand Hermann ${ }^{4} \cdot$ Pascale Gaussem $^{1} \cdot$ Tristan Mirault $^{9} \cdot$ Benjamin Terrier $^{10}$. Olivier Sanchez ${ }^{7}$. Jean-Luc Diehl ${ }^{11}$. Michaela Fontenay ${ }^{12} \cdot$ David M. Smadja $^{1}$}

1 Université de Paris, Innovative Therapies in Haemostasis, INSERM, 75006 Paris, France, Hematology Department and Biosurgical Research Lab (Carpentier Foundation), Assistance Publique Hôpitaux de Paris. Centre-Université de Paris (APHP.CUP), 20 rue Leblanc, 75015 Paris, France

2 Université de Paris, PARCC, INSERM, Emergency Department, Assistance Publique - Hôpitaux de Paris-Centre (APHP-CUP), 75015 Paris, France

3 Université de Paris, Emergency department, Assistance Publique - Hôpitaux de Paris-Centre (APHP-CUP), 75015 Paris, France

4 Université de Paris, Assistance Publique - Hôpitaux de Paris-Centre (APHP-CUP), 75015 Paris, France

5 Université de Paris, Vascular Medicine Department and Biosurgical Research Lab (Carpentier Foundation), Assistance Publique Hôpitaux de Paris-Centre (APHP-CUP), 75015 Paris, France

6 Université de Paris, Innovative Therapies in Haemostasis, INSERM, 75006 Paris, France, Curie Institute, Cytometry department, 75006 Paris, France

7 Université de Paris, Innovative Therapies in Haemostasis, INSERM, 75006 Paris, France, Respiratory Medicine
Department and Biosurgical Research Lab (Carpentier Foundation), Assistance Publique - Hôpitaux de Paris-Centre (APHP-CUP), 75015 Paris, France

8 Université de Paris, Innovative Therapies in Haemostasis, INSERM, 75006 Paris, France, Hematology Department Assistance Publique Hôpitaux de Paris.Centre-Université de Paris (APHP-CUP), 75014 Paris, France

9 Université de Paris, PARCC, INSERM, 75015 Paris, France, Vascular medicine department, Assistance Publique - Hôpitaux de Paris-Centre (APHP-CUP), 75015 Paris, France

10 Université de Paris, PARCC, INSERM U970, 75015 Paris, France, Internal Medicine Department, AH-HP-Centre Université de Paris (CUP), 75014 Paris, France

11 Université de Paris, Innovative Therapies in Haemostasis, INSERM, 75006 Paris, France, Intensive Care Unit and Biosurgical Research Lab (Carpentier Foundation), Assistance Publique - Hôpitaux de Paris-Centre (APHP-CUP), 75015 Paris, France

12 Université de Paris, Institut Cochin, INSERM, 75014 Paris, France, Hematology Department Assistance Publique Hôpitaux de Paris. Centre-Université de Paris (APHP-CUP), Cochin Hospital, 75014 Paris, France 
13 Hematology Department, Assistance Publique-Hôpitaux de Paris. Centre-Université de Paris (AP-HP.CUP), Cochin Hospital, 75014 Paris, France

14 Emergency department, Assistance Publique-Hôpitaux de Paris.Centre-Université de Paris (AP-HP.CUP), Hôtel-Dieu Hospital, 75014 Paris, France

15 Université de Paris, Institut Cochin, INSERM, 75014 Paris, France
16 Intensive Care Medicine and Reanimation Department, Assistance Publique-Hôpitaux de Paris.Centre-Université de Paris (AP-HP.CUP), Cochin Hospital, 75014 Paris, France

17 Anaesthesia, Intensive Care and Perioperative Medicine Department, GHU AP-HP, Centre - Université de Paris - Cochin Hospital, 75014 Paris, France

18 Internal Medicine Department, AP-HP.Centre - Université de Paris - Cochin Hospital, 75014 Paris, France 